\title{
Traffic flow model at fixed control signals with discrete service time distribution
}

\author{
Lucky I. Igbinosun ${ }^{1, *}$ and Sunday E.Omosigho ${ }^{2}$ \\ ${ }^{1}$ Department of Mathematics and Statistics, University of Uyo, \\ Nwaniba Road, Uyo, Akwa Ibom State, Nigeria. \\ 〈luckyigbinosun@uniuyo.edu.ng \\ ${ }^{2}$ Department of Mathematics, University of Benin, \\ Uselu, Benin City, Nigeria. \\ $\langle$ seomosigho@uniben.edu〉
}

\begin{abstract}
Most of the models of road traffic flow at fixed-cycle controlled intersection assume stationary distributions and provide steady state results. The assumption that a constant number of vehicles can leave the system during the green phase is unrealistic in real life situations. A discrete time queuing model was developed to describe the operation of traffic flow at a road intersection with fixed-cycle signalized control and to account for the randomness in the number of vehicles that can leave the system. The results show the expected queue size in the system when the traffic is light and for a busy period, respectively. For the light period, when the traffic intensity is less than one, it takes a shorter green cycle time for vehicles to clear up than during high traffic intensity (the road junction is saturated). Increasing the number of cars that can leave the junction at the turn of the green phase reduces the number of cycle times before the queue is cleared.
\end{abstract}

Key words: vehicle queue size, fixed cycle, road traffic intersection

Received: January 25, 2015; accepted: March 4, 2016; available online: March 31, 2016

DOI: $10.17535 /$ crorr.2016.0002

\section{Introduction}

Road traffic queues are waiting lines which occur whenever vehicles must wait to access a facility. An intersection may be controlled or signalized for a number of reasons, most of which relate to the safety and effective movement of conflicting vehicular and pedestrian flows through the intersection. The facility may be busy and therefore unavailable to render the required service, thus resulting in congestion. Road traffic congestion is a problem in many countries. It causes considerable costs due to unproductive time losses, accidents, air

\footnotetext{
${ }^{*}$ Corresponding author.
} 
pollution, fuel wastage, etc. The problem of road traffic congestion is worsened in many cities by the lack of real time information on traffic flows, lack of adequate data gathering facilities and a systematic methodology for optimizing road traffic flow through available road facilities. Given the cost of road traffic congestion to society, a comprehensive study of urban road network is necessary. A good understanding of vehicular traffic flow is a knowledge gap in modern societies.

Some initiatives such as staggered work hours, flextime, ramp metering, road widening, etc. have been proposed to alleviate problems associated with road traffic congestion, but the level and period of congestion in most major urban areas is increasing.

Viti and Van Zuylen [23] argue that "queue estimation at a controlled intersection is a problem in transportation engineering and operations research." Queue dynamics have been modeled as deterministic phenomena, and under rather limiting assumptions of a steady state scenario. In urban networks, these assumptions are far from being deterministic or certain. Deterministic models based on fluid theory have also been studied and demonstrated as appropriate for highly oversaturated scenarios [14]. But Viti and Van Zuylen [23] state that "these models are incapable of estimating the temporal effects that occur when signals operate near the signal capacity. Modeling these effects and their probability of occurrence becomes particularly important." The vast set of models presented in [23] indicate that there is still no clear insight into the way queues are experienced at signalized intersections.

The paper is arranged as follows: We present the problem statement in Section Two and provide a cursory review of literature relevant to the subject of study in Section Three. In Section Four, we present the discrete model for traffic flow at a road intersection with a fixed-cycle light. The results are presented in Section Five and the conclusion of the work is given in Section Six.

\section{Problem statement}

Viti and Van Zuylen [23] list, among others aspects, the shortcomings of the previous time-dependent models which include: (1) The assumption that arrivals and departures follow a specific distribution and have stationary rates. (2) The initial queue is assumed to be zero at the beginning of the evaluation period. In the analysis of queues at fixed-controlled intersections, Viti and Van Zuylen [23] however, provide a methodology that enables the capturing of dynamic and stochastic effects on queue length that originate from the variability of arrivals. Omosigho [13] however, shows that if the number of cars at the beginning of the green phase, $i$, is less or equal to the number of cars departing during the green phase, $s$, i.e. $i \leq s$, the expression, 


$$
\sum_{n=0}^{i-s} p_{n}(n<i-s), \quad i-s \leq a_{\max }
$$

given by Viti and Van Zuylen [23] fails to properly capture a situation, which in turn could lead to a zero state. Omosigho [13] enumerates the events that may lead to state zero for all $i \leq s$ and gave a corrected version as,

$$
P Q G(0)=\sum_{k=0}^{s} P 2(k)\left(\sum_{i=0}^{s-k} A 2(i)\right)
$$

where $P Q G(0)$ is the probability of having no cars in the queue at the end of the green phase, $P 2(k)$ the probability of $k$ cars in the queue at the beginning of the green phase, and A2(i) the probability of $i$ arrivals during the green phase. However, Viti and Van Zuylen [23] and Omosigho [13] assume that the number of vehicles that can leave the system during the green phase is constant. In practice, this assumption is unrealistic. In view of the limitations, we develop a discrete time-dependent model to describe the operation of traffic flow at a road intersection with fixed-cycle signalized control and to account for the randomness in the number of vehicles that can leave the system.

\section{Literature review}

We will discuss some existing works on road traffic queues at signalized intersections using queuing theory presented in this paper.

Darroch [8] presents a formal solution for the stationary distribution of queue length at a fixed-cycle traffic light for a fairly general distribution of arrivals and for a single stream of vehicle. Expressions for the expected queue length and the expected delay per vehicle were presented.

Rouphail et al. [14] analyse road traffic flow at signalized intersections and emphasizes the theory of descriptive models of traffic flow. Several models including the fluid theory approach and the steady-state queuing approach along with their various advantages and limitations are discussed. In studying or modeling traffic flow at controlled intersections, the average delay per vehicle, number of stopped vehicle, number of queued vehicles, expected delays and the average queue length are among the important performance measures that can be derived. Due to the minimal effect of the stochastic queuing approach in extremely congested conditions, the fluid theory approach is considered more appropriate to use for highly over-saturated conditions. In concluding, Rouphail et al. [14] agree that there are areas requiring further attention and research, and the assumption of uncorrelated arrivals found in most models is inadequate for describing flow. Secondly, an estimation of the initial overflow queue at a signal is inadequately understood and documented. Queuing models constrained by physical space available for queuing should also be developed. 
Van Leeuwaarden [17] considers the fixed-cycle traffic light (FCTL) queue, where vehicles arrive at an intersection controlled by a traffic light and form a queue. He presents the queue length and delay distributions by considering two arrival Poison and geometric distributions. A method for inverting the generating functions was also presented with the conclusion that the mean delay is sensitive to the stochastic properties of the arrival distribution.

Dion et al. [9] present a summary of delay models for signalized intersections and include deterministic queuing models, a shock wave delay model, steady-state stochastic delay models, and time-dependent stochastic delay models for both under-saturated and over-saturated conditions, microscopic simulation delay models. The ITEGRATION ${ }^{\mathrm{TM}}$ simulation package was used as a benchmark for other models with the conclusion that all compared delay models produce similar results for signalized intersections when traffic intensity is low. But under heavy traffic, noticeable differences occur.

Kakooza et al. [11] use a simple mathematical model (steady state $\mathrm{m} / \mathrm{m} / \mathrm{s}$ queuing model) to analyze different types of road intersections in terms of their performance and in relation to managing traffic congestion and establishing congestion for stabilizing road intersections after sufficiently longer periods of time (steady-state). The authors analyzed un-signalized and signalized intersections, and roundabouts. They obtained expressions for the steady state expected number and waiting time of vehicles stopping at a road intersection interrupted by delays. For a single lane system $(\mathrm{m} / \mathrm{m} / 1)$, Kakooza et al. [11] give an expression for the expected number of vehicles in the system as:

$$
E(X)=\frac{\lambda\left[(r+f)^{2}+\mu_{0} f\right]}{(r+f)\left[r\left(\mu_{0}-\lambda\right)-\lambda f\right]}
$$

Where, $\lambda$ is the average number of vehicles arriving at an intersection per unit time; $r$ is the rate of disappearance or clearance of the delays; $f$ is the rate of occurrence of delays; and $\mu_{0}$ denotes the service rate with no delays. For stability (avoiding an ever-increasing back-log of vehicles), they assert that the traffic intensity $\left(\rho=\lambda / \mu_{0}\right.$, for a single lane) must be less than the proportion of delay time $\left(\tau=\frac{r}{(r+f)}\right)$. The waiting time was given as:

$$
W(X)=\frac{E(X)}{\lambda}=\frac{\left[(r+f)^{2}+\mu_{0} f\right]}{(r+f)\left[r\left(\mu_{0}-\lambda\right)-\lambda f\right]}
$$

They conclude that "since congestion is as a result of heavy traffic, the best option in managing traffic congestion at an intersection would be to replace unsignalized and roundabout intersections with signalized intersections in case such intersections have heavy approaching traffic".

Their method and results are similar to those of Baykal-Gursoy et al. [5] using probability generating functions. Excess demand for road space, irregular 
occurrences such as traffic accidents, vehicle disablements, spilled loads and hazardous materials were identified by Baykal-Gursoy et al. [5] as some causes of road flow reduction.

Baykal-Gursoy and Xiao [4] consider a steady-state $\mathrm{m} / \mathrm{m} / \infty$ queuing system subjected to random interruptions of exponentially distributed durations. Total system breakdown and partial failure were investigated, and in both cases present the expected number of vehicles in the system. Under the impact of the interruption, all servers work at lower efficiency until the interruption is cleared. Varying the parameters with respect to the expected number of vehicles in the system (as given in [5]), the impact of each parameter of $\mathrm{E}(\mathrm{X})$ was presented. The steady-state performance measure (expected number of vehicles in the system) was presented for $\mathrm{m} / \mathrm{m} / 2$ and $\mathrm{m} / \mathrm{m} / 3$ in [3] where they stated that "The analysis of the $\mathrm{M} / \mathrm{MSP} / \mathrm{C}$ queue with $\mathrm{n}$ server states clearly indicates that explicit solutions for the general case would be difficult to obtain".

Van Zuylen and Viti [18] propose a method of solving the calculation of queues and delays at a signalized intersection using a Markov chain model to derive the probability distribution of queue length within a cycle time and using any initial queue length distribution. Based on the dynamics of the expectation value for queue length, the authors derive a formula for the delay in fixed time traffic control; provide an exact probabilistic formulation of the queuing process within a cycle, which enables justification of the dynamic and stochastic character of overflow queues, especially at signals that operate near capacity. The challenge, however, is in the assumption of a constant, deterministic departure rate, while no specific distribution is assumed for arrivals.

Viti and Van Zuylen [22] propose a probabilistic model for queues at a fixed and actuated controlled signalized intersection. They showed that a probabilistic modeling approach can help to explain different traffic conditions, different signal types, etc. by simply assuming a probability distribution for the number of arrivals and departures within a cycle. However, these models do not provide insight into the way queues are experienced by drivers at signalized intersections (see also [22]). Road traffic conditions are not static; this has been observed by many authors including Cherrett et al. [6]. It is therefore desirable to develop models that incorporate realistic assumptions.

Lartey [12] presents a mathematical modeling and prediction of road congestion on an urban road in Ghanaian using queuing theory based on a stochastic process and initial value problem framework. The approach describes performance measure parameters, thus predicting the increase in queue level at a signalized intersection and subsequently providing an insight into road vehicular congestion and how the occurrence of such congestion can be managed. The author links time evolution phenomena to vehicle queues at a signalized intersection to constitute an observed stochastic processes. The work presents an analysis of vehicular traffic congestion at a signalized intersection 
with the hope of gaining insight into certain performance measures. Lartey [12] asserts that the distribution of the busy period is the length (or duration) of time during which the server remains busy. The busy period is the interval from the moment of the arrival of a unit vehicle at an empty system to the moment that the channel becomes free for the first time. This, therefore, constitutes a random variable.

In [1], a discrete event simulation model was developed, verified and validated. The model was tested for different scenarios. The objective by Aljaafreh et al [1] was to determine the optimized timing parameters for traffic signal based through simulation. The author argues that "designing a real-time proactive adaptive control model for traffic will enhance the performance of traffic lights". In this paper, however, we seek to show the behavior of a signalized road traffic intersection using a simple discrete model. The model will be tested for both cases of road traffic intensities $(\rho<1$ and $\rho \geq 1)$.

Vasic [20] makes certain considerations in her Discrete Simulation Model for Heterogeneous Traffic Including Bicycles on Urban Road Networks. The heterogeneity of vehicle type was provided by allowing different cell sizes in the model, based on cells of different sizes and specific vehicle type parameters, such as maximal velocity.

Tiriolo et al. [15] present a traffic flow model, based on the cell transmission concept, with the aim of recording urban traffic dynamics taking into account complex flow interactions among lane groups at upstream of signalized intersections. The model is designed to simulate, at macroscopic level, more realistically the dynamic interaction of queues among neighboring lanes and intersections for large scale urban networks. The traditional intersection traffic model is extended to take into account some real aspects of traffic conditions, such as the proportion of turning and lane width with respect to different movements.

Asenime and Mobereola [2] evaluated traffic behavior at different peak periods at the Maryland Interchange in Metropolitan Lagos. The authors categorized predictable behavior into three periods namely: morning, inter-peak and evening peak periods. Asenime and Mobereola [2] reported that the signal level of traffic service controlled traffic flow effectively at the intersections, however, they agreed that land use factors had a negative impact on traffic flow. 


\section{Model development}

\subsection{Notation}

We will list the parameters used in this paper as follows:

Let $P_{i j}(t)$ be the probability of transition from state $i$ to state $j$ during a time interval of length $t$.

$A_{G}(k)$, the probability of $k$ arrivals during the green phase,

$P_{G}(i)$, the probability of $i$ cars in a queue at the beginning of the green phase.

$s_{k}, k=1(1) m$, the number of cars that can depart during the green phase.

$P_{k}$, the probability that $s_{k}$ cars leave the queue during the green phase.

$Q_{\max }$, the maximum number of cars that can queue at a signalized intersection.

$P G(j)$, the probability that there are $j$ cars in the queue at the end of the green phase.

$\lambda$, the arrival rate for the Poisson arrival process.

$P R(i)$, the probability of $i$ cars in the queue at the beginning of the red phase.

$A R(k)$, the probability of $k$ arrivals during the red phase.

$\operatorname{PQR}(j)$, the probability of $j$ cars in the queue at the end of the red phase. $\rho$, traffic intensity (road utilization factor)

\subsection{Model assumptions}

The arrival manner is considered Markovian and independently distributed with respect to the Poisson arrival rate $(\lambda)$. Where there are multiple lanes regulated by the same traffic light, we assume that all the lanes are treated as one, hence we have a single server case and no overtaking is allowed. We also assume that during the green phase, $s_{k}(k=1,2,3, \ldots m)$ cars can be served, but no queue is allowed ahead of the phase. The initial queue size may or may not be zero at the beginning of the green phase. A maximum number of cars $\left(Q_{\max }\right)$ is allowed at any given green phase without blocking other junctions. We further assume that the light cycle is between red and green, the amber phase is considered to be either part of the red or green phase. The cars leaving the green phase is not constant, but that the green phase (green light duration) is constant and that the traffic light is $100 \%$ effective. 


\subsection{The model}

Given that the number of cars leaving the queue during the green phase is no longer a fixed value, it is a discrete random variable. It is given the value $S_{k}$ with a probability of $P_{k}(k=1,2,3, \ldots m)$. The goal is to determine the probability of $j$ cars in the queue at the end of the green phase. To do so, the following conditions apply.

At the beginning of the green phase, we assume $i$ number of cars waiting for service; $i=0,1,2,3, \ldots$. When $i=0$, no car is queuing at the beginning of the green phase. We can have $j \geq 1$ cars in the queue at the end of the green phase if:

- $\quad S_{k}$ cars depart during the green phase.

- $i$ cars in the queue at the beginning of the green phase.

- $\quad a$ cars arrive during the green phase.

For $j$ cars at the end of the green phase, we must have:

$$
\begin{aligned}
j & =i+a-s_{k}, \\
\therefore a & =j+s_{k}-i,
\end{aligned}
$$

Hence the probability of having $j$ cars at the end of the green phase can be modeled as:

$$
P G(j)=\sum_{k=1}^{m} \sum_{i=0}^{j+s_{k}} P_{G}(i) A_{G}\left(j+s_{k}-i\right) P_{k}, \quad j=1,2,3, \ldots, Q_{\max }
$$

If $k=1$, equation (1) reduces to:

$$
\begin{aligned}
P G(j) & =\sum_{i=0}^{j+s_{1}} P_{G}(i) A_{G}\left(j+s_{1}-i\right) P_{1} \\
& =\sum_{i=0}^{j+s} P_{G}(i) A_{G}(j+s-i) .
\end{aligned}
$$

Where $s_{1}=s$ ( $s$ is const.), $P_{1}=1$. This equation is consistent with that given in [13].

Meanwhile during the red phase, the probability of having $j$ cars in the queue at the end of the phase is given by:

$$
P Q R(j)=\left\{\begin{array}{l}
\sum_{i=0}^{j} P R(i) A R(j-i), \\
0 \quad \text { otherwise }
\end{array} \quad j=i, i+1, \ldots Q_{\max }\right.
$$

Where $\operatorname{PQR}(j)$ is the probability of $j$ cars at the end of the red phase (see [13]). 
The model proposed in this paper generates time dependent probabilities of having a $j$ number of vehicles in the queue at the end of the discrete time periods (also known as regeneration points. See [7, 13]). Vehicles form a queue at the beginning of the red phase while waiting for service, and as the light turns green, cars leave the queue. Even though the cycle period is fixed, the rate of cars leaving the queue at different periods is not constant.

\section{Solution}

We used MATLAB 7.5 software to generate time dependent probabilities of having a $j$ numbers of cars in the queue at the end of the discrete time periods. For example, if the number of cars allowed to leave the system when the light turns green assumes some set of numbers, the initial number of cars in the queue is 10 . Figure 1 shows that the queue gradually disappears.

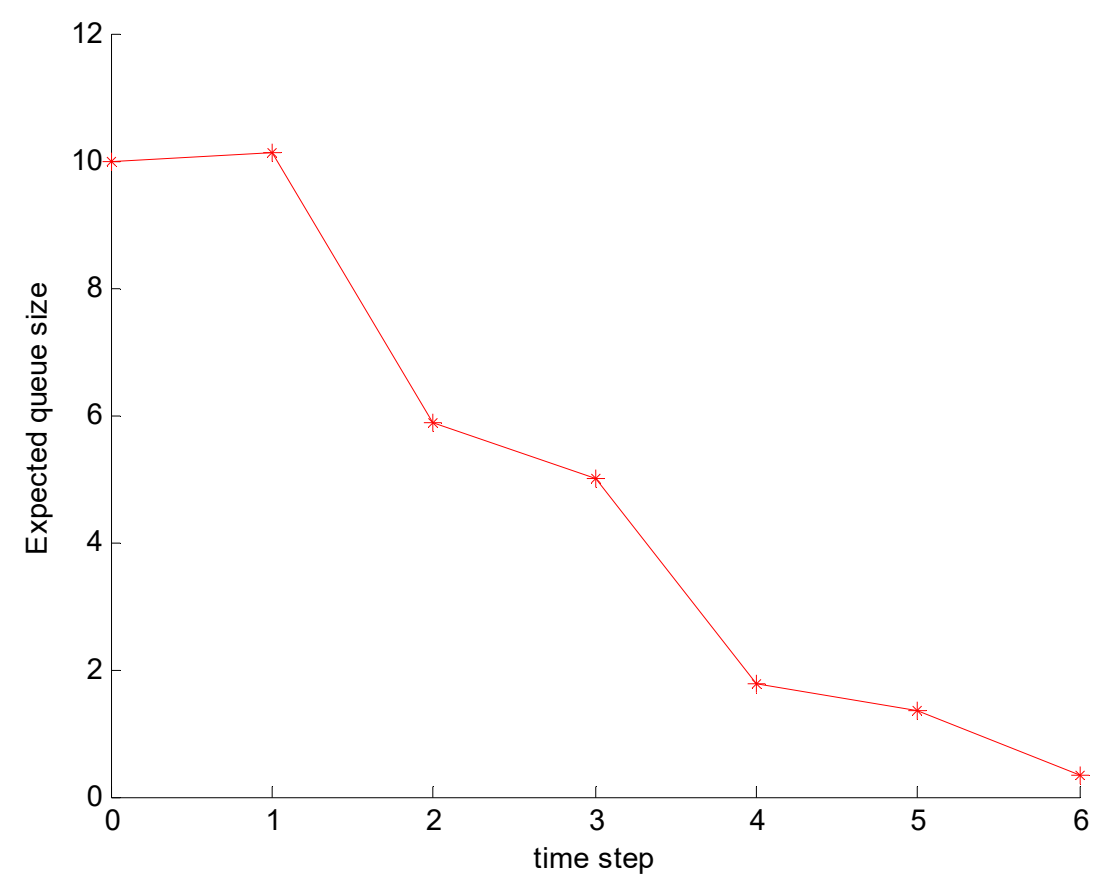

Figure 1: The expected queue length when the number of cars allowed to go during the green light is not constant. $\rho<1, Q_{\max }=50$

Observe that when the traffic intensity is low (less than one), the queue formed during the red phase is cleared during the green phase as shown in the saw-tooth-like graph in Figure 1. This result agrees with Omosigho [13] in terms of the shape, but there are differences in the expected number of vehicles in the queue. Our focus is on the time dependent behavior of the system for a short 
period of the day. For example, two to three hours is considered a short period during the day. We examine a period of free flow (when the road traffic intensity is less than one, i.e. $\rho<1)$, and a rush-hour or busy period $(\rho \geq 1)$. At both periods, we show that there is a relationship between the traffic intensity $\rho$ and $\lambda$.

When $\rho<1$, we observe that increasing the value of $\lambda$ did not affect the result. This is so because for both phases of the traffic light, the number of cars is always served (see Figures 1 and 2). Figure 2 shows the various graphs indicating the expected number of cars in the queue for various values of $\lambda$. Notice that the queues always reach zero.

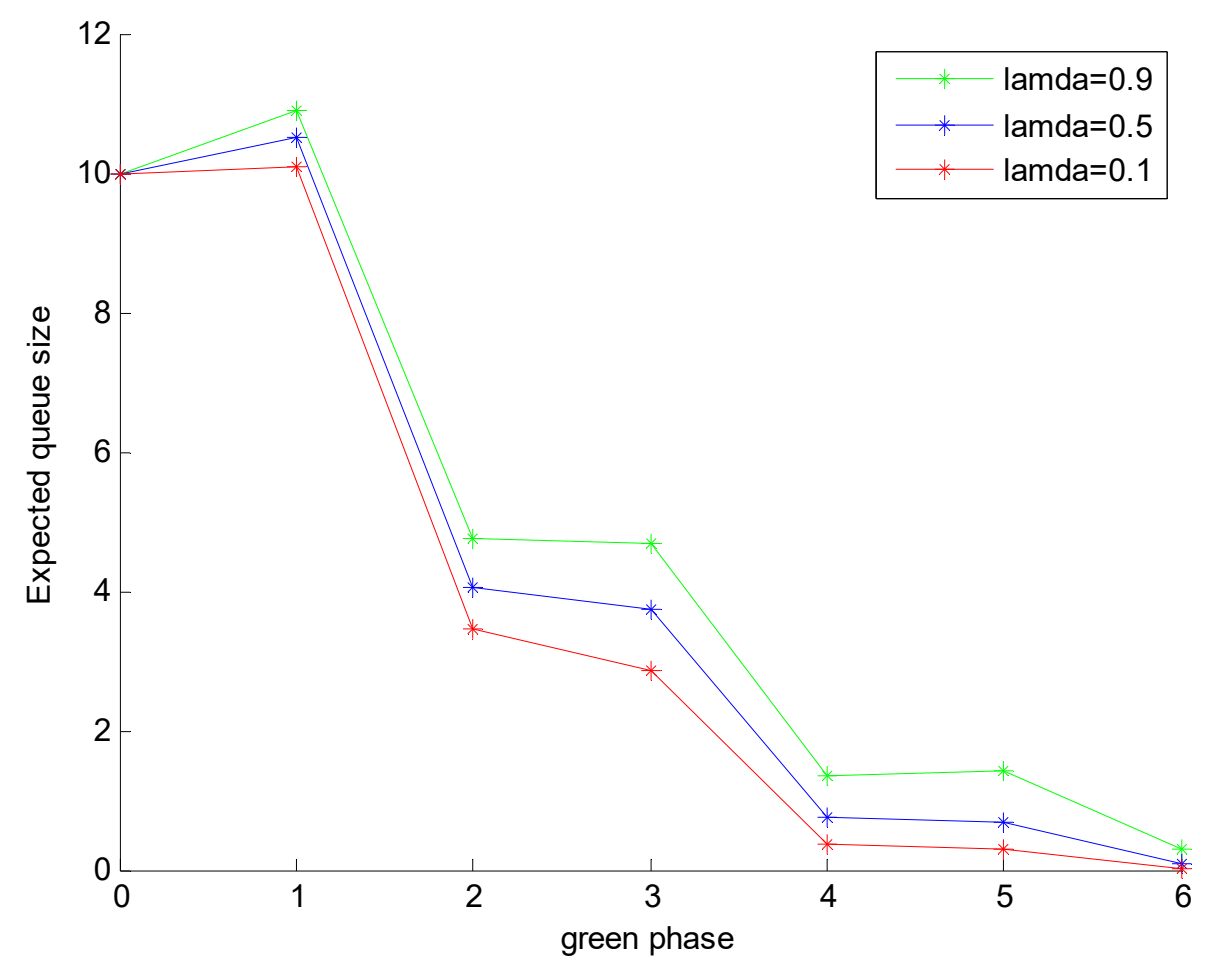

Figure 2: Expected number of vehicles in the queue for various values of $\lambda$.

$$
Q_{\max }=10,50,100,1000
$$




\begin{tabular}{|c|c|c|}
\hline Queue size $(\lambda=0.1)$ & Queue size $(\lambda=0.5)$ & Queue size $(\lambda=0.9)$ \\
\hline 10.00 & 10.00 & 10.00 \\
\hline 10.10 & 10.50 & 10.90 \\
\hline 3.47 & 4.06 & 4.77 \\
\hline 2.87 & 3.73 & 4.70 \\
\hline 0.38 & 0.75 & 1.34 \\
\hline 0.28 & 0.68 & 1.40 \\
\hline 0.00 & 0.78 & 0.28 \\
\hline
\end{tabular}

Table 1: Expected queue size at the turn of green phase

Table 1 shows the expected number of vehicles in the queue at the end of the green phase when the traffic intensity is less than one. Observe that the queue gradually vanishes.

When $\rho \geq 1$, the values of $\lambda$ and the number of vehicles in motion during the green phase becomes important. This is true because the expected number of cars in the queue will continue to grow in time if the number of vehicles permitted to head off at the onset of the green phase is small. Figure 3 demonstrates the fact that congestion results at the traffic junction. In practice, this is a problem. One way of solving this is to adjust the green phase duration to accommodate the passage of more vehicles.

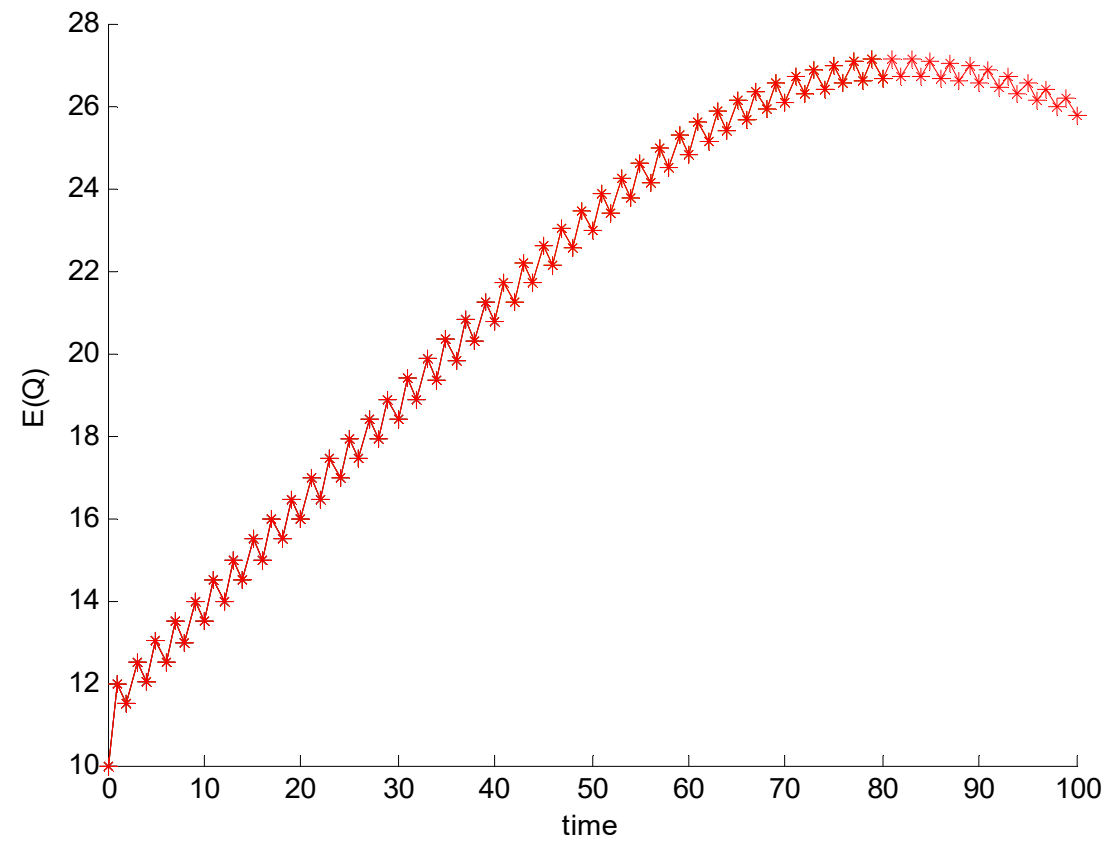

Figure 3: Expected queue size when the traffic intensity is greater than one, 


$$
s=(1,2) \lambda=2, Q_{\max }=100
$$

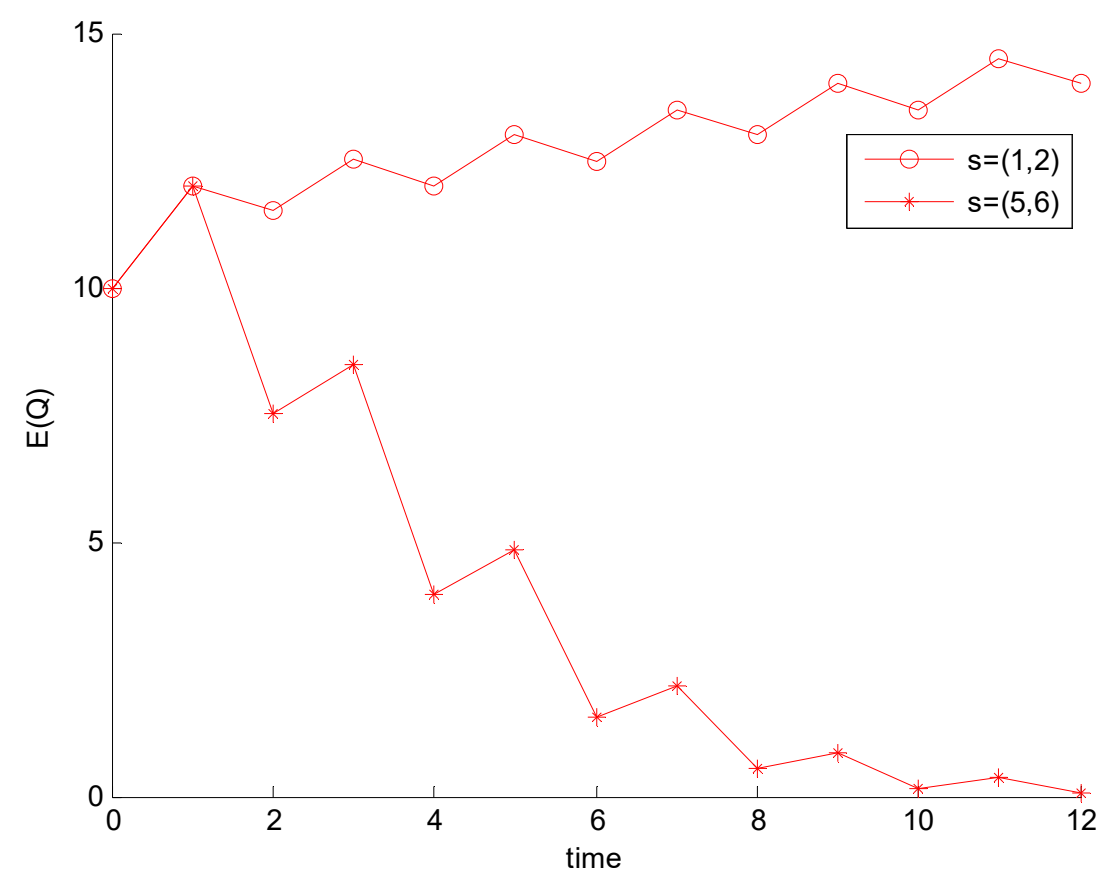

Figure 4: Expected queue size when the traffic intensity is greater than one,

$\lambda=2, Q_{\max }=100$, but with increased number of cars permitted to go during the green phase.

Figure 4 shows that increasing the number of cars permitted to go during the green phase reduces congestion (increase of the queue size). It takes longer green circle periods for the queue to clear when a very limited number of cars are permitted to go during the green phase.

\section{Conclusion}

A discrete time model of traffic flow at signalized intersections has been presented in this paper, where the proposed model considers the case when the number of cars departing the queue at the turn of the green light is not constant and therefore assumes a probability distribution. Constant departure rates have been presented in the literature (see $[23,13])$. The results presented show that transient measures of performance (expected queue length) at different cycle times can be achieved. They also show that for very small value(s) of traffic intensity, there exists a direct relationship between the departure size and the utility function of the traffic junction. Allowing a very small number(s) of cars 
to depart at the turn of the green cycle phase reveals that traffic build up occurs over longer cycle times in order to clear when compared to a greater number(s) of departing cars. When the light turns green, and cars depart from the queue, the study has shown that the number of cars leaving the service point may not necessarily be constant. Observation shows, however, that the nature and type of vehicles (for example, it takes a longer time for a truck vehicle to leave the queue compared to a car) are a factor in determining the number of vehicles that can leave the queue at the turn of the green phase. Motor bikes also struggle to receive service at the start of green phase, [13]. Incorporating these into the model assumption can be an interesting formulation in future research. Results from the model in this paper will help road traffic engineers in building of road traffic lights to function at various green phase times in order to indicate the location and density of cars on a particular road.

\section{References}

[1] Aljaafreh, A., Al-Oudat, N. and Saleh, M. (2014). Adaptive traffic-signal control using discrete event simulation model. International Journal of Computer Applications, 101(12), 7-12.

[2] Asenime, C. and Mobereola, D. (2015). Traffic behaviour at a signalised intersection in metropolitan Lagos. American Journal of Social Issues and Humanities, 5(2), 453-461.

[3] Baykal-Gursoy. M. and Duan, Z. (2006). M/M/C Queues with Markov modulated service processes. Value Tools, October 14, 2006. Pisa, Italy.

[4] Baykal-Gursoy, M. and Xiao, W. (2004). Stochastic decomposition in $M / M / \infty$ Queues with Markov modulated service rates. Queueing Systems, 48(1), 75-88.

[5] Baykal-Gursoy, M., Xiao, W. and Ozbay, K. (2009). Modeling traffic flow interrupted by incidents. European Journal of Operational Research, 195(1), 127138 .

[6] Cherrett T., McLeod F., Bell H. and McDonald M. (2002). Journey time estimation using single inductive loop detectors on non-signalized links. Journal of Operations Research Society, 53(6), 610-619.

[7] Cox, D. R. and Smith, W. L (1963). Queues. Chapman and Hall, London.

[8] Darroch, J. N., Newell, G. F. and Morris, R. W. J. (1964). Queues for vehicleactuated traffic light. Operations Research, 12(6), 882-895.

[9] Dion, F., Hesham, R. and Youn-Soo, K. (2004). Comparison of delay estimates at under-saturated and over-saturated pre-timed signalized intersections. Transportation Research Part B, 38(2), 99-122.

[10] Igbinosun, L. I. (2002). Road traffic queues and delays at road junction: A case study. Unpublished M.Sc Project submitted to the post graduate school, University of Benin, Benin City, Nigeria.

[11] Kakooza, R., Luboobi, L. S. and Mugisha, J. Y. T. (2005) Modeling traffic flow and management at un-signalized, signalized and roundabout road intersections. Journal of Mathematics and Statistics, 1(3), 194-202. 
[12] Lartey, J. D. (2014). Predicting traffic congestion: A queuing perspective. Open Journal of Modelling and Simulation, 2, 57-66.

[13] Omosigho, S. E. (2011). Discrete time queuing model for queues at fixed control signalized intersection. International Conference on Scientific Computing. Abuja, August $14^{\text {th }}-20^{\text {th }}$.

[14] Rouphail, N., Tarko, A. and Li, J. (2000). Traffic flow at signalized intersections, Chapter 9 of the Update of Transportation Research Board Special Report 165, 'Traffic flow theory', 1998. Available at http://wwweta.ornl.gov/cta/research/trb/tft.html [Accessed on May 12, 2015].

[15] Tiriolo, M., Adacher, L. and Cipriani, E. (2014). An urban traffic flow model to capture complex flow interactions among lane groups for signalized intersections. Procedia - Social and Behavioral Sciences 111, 839-848.

[16] Van Hinsbergen, C. P. I. and Van Lint, J. W. C. (2008). Bayesian combination of travel time prediction models. Transportation Research Record, 2064, 73-80.

[17] Van Leeuwaarden, J. S. H. (2006). Delay analysis for the fixed-cycle traffic-light queue. Transportation Science, 40(2), 189-199.

[18] Van Zuylen, H. J. and Viti, F. (2006). Queues at controlled intersections: The old theory revisited. In: Proceedings of the 2006 IEEE Intelligent Transportation Systems Conference, Toronto, Canada, September $17^{\text {th }}-20^{\text {th }}$.

[19] Van Zuylen, H. J. and Viti, F. (2003). Uncertainty and the dynamics of queues at controlled intersections. Proceedings CTS-IFAC Conference, 6-8 August; Tokyo. Elsevier.

[20] Vasci, J. (2014). A discrete simulation model for heterogeneous traffic including bicycles on urban road networks. Unpublished PhD Thesis submitted to School of Computing, Faculty of Engineering and Computing, Dublin City University. Available at: http://www-doras.dcu.ie/19765/1/jv_thesis.pdf [Accessed on December 16, 2015].

[21] Viti, F. and Van Zuylen, H. J. (2004). Modeling queues at signalized intersections. Transportation Research Record 1883, 68-77.

[22] Viti, F. and Van Zuylen, H. J. (2009). The dynamics and the uncertainty of queues at fixed and actuated controls: A probabilistic approach. Journal of Intelligent Transportation Systems, 13(1), 39-51.

[23] Viti, F. and Van Zuylen, H. J. (2010). Probabilistic models for queues at fixed control signals. Transportation Research Part B, 44(1), 120-135. 